\title{
SPECIFIC UPTAKE OF NOREPINEPHRINE AND DOPAMINE BY HOMOGENATES OF RAT CEREBRAL CORTEX AFTER LOCUS CERULEUS LESION ${ }^{1}$
}

\author{
WILLIAM J. LOGAN ${ }^{2}$ AND SAMI I. HARIK \\ Division of Neurology, Departments of Pedialrics and Medicine, University of Toronto, The Hospital for Sick Children, \\ Toronto, Ontario, Canada M5G $1 X 8$ and Department of Neurology, University of Miami School of Medicine,
} Miami, Florida 33101

Received June 26, 1981; Revised November 9, 1981; Accepted November 13, 1981

\begin{abstract}
Unilateral injection of 6-hydroxydopamine into the locus ceruleus led to long lasting depletion of norepinephrine in the ipsilateral parietal cerebral cortex of the rat up to 8 weeks after injection. The uptake of both norepinephrine and dopamine by homogenates of parietal cortex was decreased markedly on the ipsilateral, but not on the contralateral, side. The uptake of four other neurotransmitter candidates or precursors was unaffected by the lesion. Desipramine (DMI) markedly inhibited the uptake of both norepinephrine and dopamine by cortical preparations from the contralateral side and from control rats, but this effect was not seen in preparations from the norepinephrinedepleted, ipsilateral cortex. The decreased DMI-sensitive uptake of norepinephrine or dopamine was highly correlated with decreased norepinephrine in the ipsilateral cortex. These results suggest that the specific uptake of norepinephrine and dopamine is a valid marker of noradrenergic innervation of the parietal cortex and that dopamine seems to be accumulated primarily by norepinephrine terminals in this region. Two and 8 weeks after locus ceruleus lesion, a DMIinsensitive, benztropine-sensitive dopamine uptake becomes apparent in ipsilateral cortical preparations. This may represent proliferation or increased function of dopaminergic cortical projections, compensating for noradrenergic denervation.
\end{abstract}

Lesion of the locus ceruleus $(\mathrm{LC})^{4}$ induced by the local stereotaxic injection of 6-hydroxydopamine (6-OHDA) results in a marked and sustained decrease in tissue levels of norepinephrine (NE) in the ipsilateral cerebral cortex (Harik et al., 1981). This is associated with increased density of $\beta$-adrenergic receptors (Sharma et al., 1979, 1981) and abnormalities of cerebral oxidative metabolism when the cerebral cortex is stimulated to heightened activity (Harik et al., 1979; LaManna et al., 1981). These receptor and metabolic alterations in the NE-depleted

\footnotetext{
${ }^{1}$ This work was supported by the Grotto Cerebral Palsy Foundation, Inc., Toronto and by Public Health Service Grants NS 16617 and NS 05820. We wish to thank E. Gagalang for expert technical assistance.

${ }^{2}$ To whom correspondence should be addressed at Division of Neurology, The Hospital for Sick Children, 555 University Avenue, Toronto, Ontario, Canada M5G 1X8.

${ }^{3}$ Present address: Department of Neurology, Case Western Reserve University, Cleveland, $\mathrm{OH} 44106$.

${ }^{4}$ The abbreviations used are: BZT, benztropine; CHOL, choline; DA, dopamine; DMI, desipramine; GABA, $\gamma$-aminobutyric acid; GLUT, Lglutamic acid; 5 -HT, serotonin; LC, locus ceruleus; NE, norepinephrine; NT, neurotransmitter; 6-OHDA, 6-hydroxydopamine.
}

cortex reach their maximum 2 weeks after LC lesion but return to normal within 4 to 8 weeks (Harik et al., 1981), thus indicating the presence of compensatory mechanisms that enable the cerebral cortex to adjust to chronic noradrenergic denervation. The mechanisms mediating this recovery are not known. One possibility is regeneration of the lesioned noradrenergic system. Alternatively, recovery may be due to alterations in other central neurotransmitter systems.

To determine whether such recovery is mediated by the sprouting of new noradrenergic or other neurotransmitter terminals, the high affinity uptake of NE and other putative neurotransmitters or neurotransmitter precursors (NTs) was used in this study as a marker of the synaptic terminals subserving these neurotransmitter systems. Uptake was evaluated in homogenates of ipsilateral (NE-depleted) and contralateral (control) cerebral cortex of rats with a unilateral LC lesion studied at 2 and 8 weeks after the lesion. We find no evidence for noradrenergic reinnervation but present data suggesting the emergence of a dopamine (DA) uptake process. Proliferations of dopaminergic terminals may be the compensatory mechanism underlying recovery of function after 
chronic LC lesion. A preliminary communication of these results has been presented (Logan and Harik, 1981).

\section{Materials and Methods}

Animals. Adult male Wistar rats weighing 175 to 225 $\mathrm{gm}$ at the time of LC lesion were used in all experiments. Rats were housed (four per cage) under diurnal light conditions with unlimited access to food and water. They were allowed a minimum of 5 days of acclimation before LC lesions were undertaken.

LC lesion. The $\mathrm{LC}$ was lesioned by the local stereotaxic microinjection of $6 \mu \mathrm{g}$ of 6-OHDA base dissolved in $3 \mu \mathrm{l}$ of $0.9 \% \mathrm{NaCl}$ solution containing $1 \mathrm{mg} / \mathrm{ml}$ of ascorbic acid and adjusted to a $\mathrm{pH}$ of 5.5. Details of the lesion procedure have been reported previously (LaManna et al., 1981; Sharma et al., 1981). LC lesions were made unilaterally with equal distribution between the left and right sides.

The uptakes of $l$-norepinephrine (NE), dopamine (DA), serotonin (5-HT), choline (CHOL), $\gamma$-aminobutyric acid (GABA), and L-glutamic acid (GLUT) were assayed using techniques similar to those previously described (Logan and Snyder, 1972; Logan and Swanson, 1979). Two or 8 weeks after LC lesion, rats were decapitated and cortical samples were taken from the parietal region of both sides. Part of the tissue was frozen immediately on dry ice for $\mathrm{NE}$ assay. The remainder was homogenized in $9 \mathrm{vol}$ of $0.3 \mathrm{M}$ sucrose using a motor-driven Teflon pestle and glass homogenizer. All procedures were performed at 0 to $4^{\circ} \mathrm{C}$ unless otherwise stated. The homogenate was diluted further with $0.3 \mathrm{M}$ sucrose and centrifuged at $17,000 \times g$ for $10 \mathrm{~min}$. The pellet was resuspended in Tris-buffered Krebs' solution ( $\mathrm{pH} 7.4$ ), and aliquots were used for uptake studies in quadruplicate. The uptake incubation medium $(0.5 \mathrm{ml})$ contained 0.1 $\mu \mathrm{M}$ tritium-labeled NT ( 25 to $70 \mathrm{Ci} / \mathrm{mmol}), 0.1 \mathrm{~mm}$ ascorbic acid, and 1 to $2 \mathrm{mg}$ of tissue homogenate. Incubations were performed for $5 \mathrm{~min}$ at $25^{\circ} \mathrm{C}$, and the reaction was terminated by transfer to an ice bath and the rapid addition of $2.5 \mathrm{ml}$ of ice cold $0.9 \% \mathrm{NaCl}$ solution containing $0.1 \mathrm{mM}$ unlabeled NT. Samples were centrifuged at $17,000 \times g$ for $10 \mathrm{~min}$ and the supernatant was discarded. The pellets were washed with $3 \mathrm{ml}$ of $0.9 \%$ $\mathrm{NaCl}$, dried, and transferred to a scintillation vial. Radioactivity was extracted into scintillation fluid and assayed in a liquid scintillation spectrometer.

The amount of NT accumulated by the tissue was estimated from the radioactivity in the pellet and was expressed as picomoles per gm of tissue per $5 \mathrm{~min}$. Blank values consisted of counts accumulated by the pellet in replicate samples containing saturating amounts of unlabeled NT $(0.5 \mathrm{~mm}$ for NE, DA, and 5-HT and $5 \mathrm{~mm}$ for GABA, GLUT, and CHOL). Under these conditions, the uptake of NT was mediated primarily by the high affinity uptake systems for each of the NTs studied.

To determine the specificity of catecholamine uptake by NE and DA systems, incubations were performed in the presence and absence of $0.5 \mu \mathrm{M}$ desipramine (DMI) and $1 \mu \mathrm{M}$ of benztropine (BZT), respectively (Horn et al., 1971; Tassin et al., 1974). The effectiveness of these concentrations of DMI and BZT was confirmed by separate experiments on homogenates of the cerebellum and striatum of control rats. The cerebellum was chosen to represent neural tissue with NE innervation but devoid of DA terminals, while the striatum represented neural tissue with a high density of DA terminals and little NE innervation. The difference in uptake found in the presence and absence of DMI represents the DMI-sensitive uptake which is considered to be specific for the noradrenergic system (Table I). The addition of BZT (DMI + BZT) further inhibits that portion of the uptake which is considered to be specific for the dopaminergic system (Table I). Thus, total minus DMI uptake = DMI-sensitive uptake and estimates NE-specific uptake; and DMI minus $(\mathrm{DMI}+\mathrm{BZT})$ uptake $=$ DMI-insensitive, BZTsensitive uptake and estimates DA-specific uptake.

$N E$ assay. Cortical samples were weighed in the frozen state and homogenized in $50 \mathrm{vol}$ of cold $0.1 \mathrm{~N}$ perchloric

\section{TABLE I}

Norepinephrine and dopamine uptake by homogenates of cerebellum and striatum of control rats

The values denote mean values \pm SEM of four observations. The final concentrations of DMI and B7T were 0.5 and $1 \mu \mathrm{M}$, respectively.

\begin{tabular}{|c|c|c|c|c|}
\hline \multirow{2}{*}{ Conditions } & \multicolumn{2}{|c|}{ NE Uptake } & \multicolumn{2}{|c|}{ DA Uptake } \\
\hline & Cerebellum & Striatum & Cerebellum & Striatum \\
\hline & \multicolumn{2}{|c|}{$\mathrm{pmol} / \mathrm{gm} / 5 \mathrm{~min}$} & \multicolumn{2}{|c|}{$\mathrm{pmol} / \mathrm{gm} / 5 \mathrm{~min}$} \\
\hline Total & $91 \pm 1$ & $181 \pm 9$ & $145 \pm 4$ & $835 \pm 60$ \\
\hline DMI & $27 \pm 2$ & $185 \pm 15$ & $48 \pm 5$ & $855 \pm 59$ \\
\hline $\mathrm{DMI}+\mathrm{BZT}$ & $29 \pm 2$ & $35 \pm 2$ & $44 \pm 2$ & $100 \pm 14$ \\
\hline
\end{tabular}

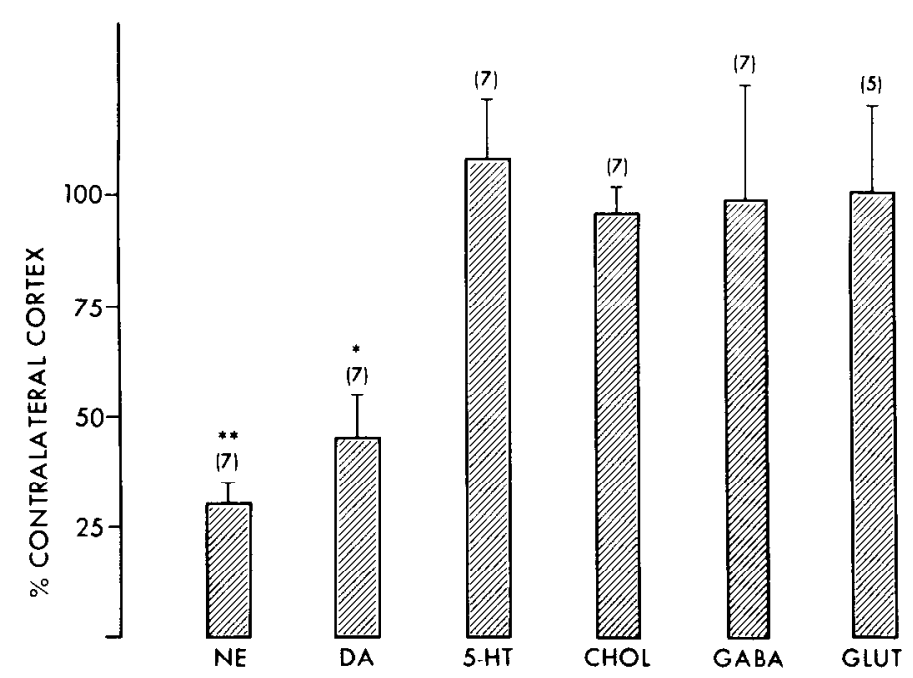

Figure 1. Uptake of $l$-norepinephrine, dopamine, serotonin, choline, $\gamma$-aminobutyric acid, and L-glutamic acid by homogenates of rat parietal cerebral cortex ipsilateral to a unilateral locus ceruleus lesion expressed as a percentage of the uptake by preparations of the contralateral cortex. The details of the uptake assay are described under "Materials and Methods." The results denote the mean values of total uptake minus a saturated blank value and are expressed in picomoles per gm of wet weight tissue per $5 \mathrm{~min}$. The number of observations is indicated in parentheses above the appropriate column. Significant differences between the two hemispheres were found only for NE and DA. ${ }^{*} p<0.01$ and ${ }^{* *} p<0.001$ by the paired $t$ test (two tailed). The experiment was performed 2 weeks after LC lesion. Tissue levels of NE were $290 \pm 19$ and $24 \pm 5$ (mean \pm SEM) $\mathrm{ng} / \mathrm{gm}$ of tissue in the contralateral and ipsilateral cortices. Similar results were obtained 8 weeks after LC lesion. 
acid. Portions of supernatant were assayed for NE in duplicate by the enzymatic-isotopic method of Henry et al. (1975).

Analysis of data. The efficacy of the LC lesion was assessed by the degree of NE depletion that it produced in the ipsilateral cerebral cortex. LC lesions were considered successful when NE was depleted by $70 \%$ or more of the NE content of the contralateral cerebral cortex. Except for the results shown in Figure 2, only data obtained from successfully lesioned rats were used in subsequent analyses. Data from the NE-depleted ipsilateral cortex was compared to that from the contralateral cortex by the paired Student's $t$ test (two tailed). Differences were considered significant at $p<0.05$.

\section{Results}

Two weeks after $\mathrm{LC}$ lesion, there was marked reduction in ipsilateral cerebral cortical NE and a corresponding decrease in the uptake of NE and DA (Fig. 1). There were no significant differences in the uptake of $5-\mathrm{HT}$, GABA, GLUT, and CHOL between the ipsilateral and contralateral sides. Similar results were obtained 8 weeks after LC lesion (data not shown).

Since NE and DA are mutual substrates for the high affinity uptake process of both noradrenergic and dopaminergic systems (Table I), specific inhibitors were used to dissect the relative contribution of each catecholamine system to the total uptake. DMI in a concentration of 0.5 $\mu \mathrm{M}$ inhibited uptake into $\mathrm{NE}$ terminals only. The addition of BZT $(1 \mu \mathrm{M})$ to DMI produced no additional inhibition of uptake by NE terminals but profoundly inhibited uptake by DA terminals (Table I).

The results of $\mathrm{NE}$ and $\mathrm{DA}$ uptake studies done in a separate series of experiments using DMI and BZT are shown in Tables II and III. Two weeks after LC lesion, total uptake of NE and DA was decreased by about $50 \%$ on the lesioned side. DMI markedly decreased uptake in the contralateral cortex but had little effect on preparations from the lesioned side. This was expected since DMI prevents uptake into NE terminals (Table I). Thus, the DMI-sensitive uptake estimates the specific uptake of NE or DA into noradrenergic terminals. Using this criterion, specific noradrenergic uptake was virtually abolished in the ipsilateral cortex (Table II). The magnitude of the decreased DMI-sensitive uptake of NE and DA in the ipsilateral cortex corresponds well with tissue NE depletion (Table II). Similar results were obtained 8 weeks after LC lesion (Table III). Thus, there is no evidence of recovery of the specific uptake of NE in the ipsilateral cerebral cortex.

The correlation between the magnitude of NE depletion and that of decreased DMI-sensitive uptake of both NE and DA in the ipsilateral cerebral cortex suggests that such specific uptake is a good indicator of the extent of the LC lesion and the resultant degeneration of cortical noradrenergic fibers and terminals. To examine this possibility further, NE depletion of the ipsilateral cortex was compared to the decrease in the DMI-sensitive uptake of $\mathrm{NE}$ and DA in a group of rats in which the success of the LC lesion in depleting cortical NE varied widely. Results of this correlation are shown in Figure 2. The correlation coefficients for NE content with NE and DA uptake were 0.84 and 0.91 , respectively.

In the presence of DMI, NE uptake was similar in the contralateral and ipsilateral cortices at 2 and 8 weeks after unilateral LC lesion. Thus, LC lesion has no effect on the DMI-insensitive uptake of NE. On the other hand, the DMI-insensitive DA uptake is increased in the ipsi-

TABLE II

Norepinephrine and dopamine uptake 2 weeks after unilateral locus ceruleus lesion

The values denote the means \pm SEM of four or five separate experiments. The final concentration of DMI was $0.5 \mu \mathrm{M}$.

\begin{tabular}{|c|c|c|c|c|c|c|c|}
\hline & \multirow{2}{*}{ NE Content } & \multicolumn{3}{|c|}{ NE Uptake } & \multicolumn{3}{|c|}{ DA Uptake } \\
\hline & & Total & DMI & Total - DMI & Total & DMI & Total - DMI \\
\hline & $n g / g m$ & \multicolumn{3}{|c|}{$\mathrm{pmol} / \mathrm{gm} / 5 \mathrm{~min}$} & \multicolumn{3}{|c|}{$\mathrm{pmol} / \mathrm{gm} / 5 \mathrm{~min}$} \\
\hline Percent change & -96 & -44 & 11 & -86 & -55 & 35 & -100 \\
\hline
\end{tabular}

" $p<0.005$ for the difference between contralateral and ipsilateral cortex.

${ }^{b} p<0.01$ for the difference between contralateral and ipsilateral cortex.

" $p<0.02$ for the difference between contralateral and ipsilateral cortex.

TABLE III

Norepinephrine and dopamine uptake 8 weeks after unilateral locus ceruleus lesion

The values denote the means \pm SEM of four or five separate experiments. The final concentration of DMI was $0.5 \mu \mathrm{M}$.

\begin{tabular}{|c|c|c|c|c|c|c|c|}
\hline & \multirow{2}{*}{ NE Content } & \multicolumn{3}{|c|}{ NE Uptake } & \multicolumn{3}{|c|}{ DA Uptake } \\
\hline & & Total & DMI & Total - DMI & Total & DMI & Total - UMI \\
\hline & $n g / g m$ & \multicolumn{3}{|c|}{$\mathrm{pmol} / \mathrm{gm} / 5 \mathrm{~min}$} & \multicolumn{3}{|c|}{$\mathrm{pmol} / \mathrm{gm} / 5 \mathrm{~min}$} \\
\hline Contralateral cortex & $313 \pm 34$ & $52 \pm 9$ & $20 \pm 4$ & $32 \pm 8$ & $92 \perp 19$ & $37 \pm 8$ & $55 \pm 19$ \\
\hline Ipsilateral cortex & $47 \pm 19^{a}$ & $27 \pm 6^{a}$ & $20 \pm 4$ & $7 \pm 2^{b}$ & $48 \pm 11^{h}$ & $48 \pm 12^{r}$ & $0 \pm 5^{d}$ \\
\hline Percent change & -85 & -48 & 0 & -79 & -48 & 30 & -100 \\
\hline
\end{tabular}

\footnotetext{
${ }^{\prime \prime} p<0.02$ for the difference between contralateral and ipsilateral cortex.

${ }^{s} p<0.02$ for the difference between contralateral and ipsilateral cortex.

$p<0.06$ for the difference between contralateral and ipsilateral cortex.

$" p<0.05$ for the difference between contralateral and ipsilateral cortex.
} 


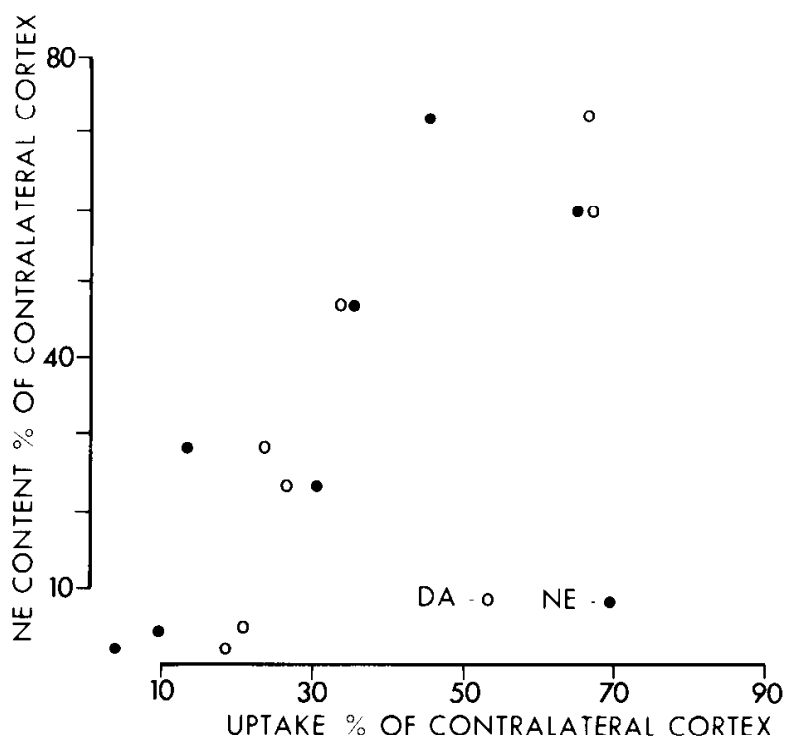

Figure 2. Norepinephrine in the parietal cerebral cortex ipsilateral to a locus ceruleus lesion expressed as a percentage of NE content of the contralateral parietal cerebral cortex is plotted against the DMI-sensitive uptake of $l$-norepinephrine and dopamine by homogenates of the ipsilateral parietal cerebral cortex also expressed as a percentage of uptake by preparations from the contralateral side. The details of the assays are described under "Materials and Methods." The efficacy of the LC lesions varied widely. DMI-sensitive uptake represents total uptake minus uptake in the presence of $0.5 \mu \mathrm{M}$ DMI. The correlation coefficient for $\mathrm{NE}$ content versus $\mathrm{NE}$ uptake was 0.84 ( $p<0.02$; two-tailed test) and for NE content versus DA uptake, it was 0.91 ( $p<0.01$; two-tailed test).

TABLE IV

Dopamine uptake 2 and 8 weeks after unilateral locus ceruleus lesion

The values denote the means \pm SEM of five separate experiments. The final concentrations of DMI and BZT were 0.5 and $1 \mu \mathrm{M}$, respectively.

\begin{tabular}{|c|c|c|c|c|}
\hline & \multicolumn{2}{|c|}{2 Weeks } & \multicolumn{2}{|c|}{8 Weeks } \\
\hline & $\begin{array}{l}\text { Contralateral } \\
\text { Cortex }\end{array}$ & $\begin{array}{l}\text { Ipsilateral } \\
\text { Cortex }\end{array}$ & $\begin{array}{l}\text { Contralateral } \\
\text { Cortex }\end{array}$ & $\begin{array}{l}\text { Ipsilateral } \\
\text { Cortex }\end{array}$ \\
\hline & \multicolumn{2}{|c|}{$\mathrm{pmol} / \mathrm{gm} / 5 \mathrm{~min}$} & \multicolumn{2}{|c|}{$\mathrm{pmol} / \mathrm{gm} / 5 \mathrm{~min}$} \\
\hline DMI & $43 \pm 8$ & $58 \pm 10$ & $37 \pm 8$ & $48 \pm 12$ \\
\hline $\mathrm{DMI}+\mathrm{BZT}$ & $44 \pm 8$ & $44 \pm 8^{a}$ & $29 \pm 4$ & $30 \pm 7^{b}$ \\
\hline Percent change & 2 & -24 & -22 & -38 \\
\hline
\end{tabular}

" $p<0.05$ for the difference between DMI and DMI + BZT.

${ }^{\circ} p<0.02$ for the difference between DMI and DMI + BZT.

lateral cerebral cortex preparations at 2 weeks $(p<0.02)$ and 8 weeks $(p=0.06)$ after lesion. This finding suggests an increased uptake of DA into non-noradrenergic elements in the ipsilateral cortex of rats with a LC lesion. To determine whether these non-noradrenergic elements are at least in part dopaminergic in origin, results of DA uptake in the presence of DMI were compared to those in the presence of DMI plus BZT (Table IV). There is a significant difference in the BZT-sensitive DA uptake in the ipsilateral cortex at 2 weeks $(p<0.05)$ and 8 weeks $(p<0.02)$ after LC lesion. This demonstrates that the increased DMI-insensitive DA uptake in the ipsilateral cortex is BZT sensitive and probably represents specific uptake into dopaminergic elements. BZT had no effect on DA uptake in preparations of the contralateral cortex.

\section{Discussion}

The LC, a paired brainstem nucleus, is the origin of most, if not all, noradrenergic innervation of the cerebral cortex. The ceruleocortical pathways are predominantly ipsilateral (Lindvall et al., 1974; I Iarik et al., 1981). LC lesion results in persistent depletion of $\mathrm{NE}$ in the ipsilateral cortex but transient effects on several other cortical parameters, such as the density of $\beta$-adrenergic receptors, the activity of isoproterenol-stimulated adenylate cyclase, and oxidative metabolism (Harik et al., 1981). The transient nature of these effects, despite continued NE depletion, could be due to noradrenergic regeneration or the sprouting of remaining NE neurons or may be due to compensatory changes in other neurotransmitter systems. Regeneration of ipsilateral NE projections is unlikely since 6-OHDA injection into the LC destroys the neurons of origin of the NE system which project to the cortex. Moreover, there is no recovery of the NE content of the ipsilateral cerebral cortex. It is still conceivable that recovery of function could occur without an accompanying increase in steady state levels of NE if the turnover rate of $\mathrm{NE}$ is increased. Alternatively, other neurotransmitters in cerebral cortex might be altered by the chronic noradrenergic denervation.

The present study utilizes uptake of neurotransmitter candidates or precursors as markers of neurotransmitter systems (Kuhar, 1973). Such uptake is altered by destruction of specific neuronal pathways (Zigmond et al., 1971; Kuhar et al., 1972, 1973; Tassin et al., 1974; Logan, 1977; Storm-Mathisen and Guldberg, 1974; Kellar et al., 1977; Storm-Mathisen, 1977). As was expected, unilateral LC lesion caused marked depletion of ipsilateral cortical NE and a concomitant decreased uptake of $\mathrm{NE}$ and DA. Uptake of other NTs appeared to be unaffected by LC lesion (Fig. 1).

Uptake of both NE and DA by contralateral and control cerebral cortex preparations is decreased by DMI, a specific inhibitor of the noradrenergic uptake system. After LC lesion, the decreased uptake of NE and DA in the ipsilateral cortex is not affected by DMI (Tables II and III). The DMI sensitivity of the NE and DA uptake in the contralateral cortex resembles the uptake by noradrenergic, but not dopaminergic, elements (Table I). This was expected because the parietal region of cortex used in these studies has little or no DA innervation (Lindvall et al., 1974). Several other observations support the conclusion that DA is accumulated primarily by $\mathrm{NE}$ uptake sites in these cortical preparations: (i) NE and DA are mutual substrates for their respective uptake systems (Table I; Snyder and Coyle, 1969). (ii) DA uptake in parietal cortex is decreased by either ipsilateral LC lesion or DMI (Tables II and III). (iii) The effect of DMI is abolished by LC lesion. The observation that DA is better than $\mathrm{NE}$ as a substrate for noradrenergic uptake systems is consistent with previous observations (Snyder and Coyle, 1969).

The decrease in total NE and DA uptake after LC lesion could be attributed to the change in the DMIsensitive portion of this uptake which correlated well with the decreased cortical NE content (Fig. 2). Thus, 
DMI-sensitive uptake of either NE or DA can serve as a marker of the noradrenergic system in the cortex and may be used to estimate the degree of $\mathrm{NE}$ depletion after LC lesions.

Following LC lesion, DA uptake in the presence of DMI (DMI-insensitive uptake) was increased in the ipsilateral cortex, but not in the contralateral cortex, at both 2 and 8 weeks after the lesion (Table IV), suggesting the emergence of uptake sites which are not noradrenergic in nature. That this increase in the DMI-insensitive uptake was sensitive to BZT indicates that these new sites resemble dopaminergic elements in their pharmacologic characteristics. It is possible that at least one of the compensatory changes that take place in the cerebral cortex after chronic noradrenergic denervation by LC lesion is the proliferation of dopaminergic endings to fill some of the spaces vacated by the degenerating noradrenergic terminals. Conceivably, these could arise from the dopaminergic innervation of adjacent cortical areas. Observations of increased dopamine content in ipsilateral cortex after LC lesion are consistent with this interpretation (Berger el al., 1974; Emson and Koob, 1978).

Recently, Acheson and colleagues (Acheson and Zigmond, 1981; Acheson et al., 1980) reported an initial decrease and a subsequent recovery of tyrosine hydroxylase activity in rat hippocampus after intraventricular injections of 6-OHDA. It is unlikely that this was due to regeneration of noradrenergic terminals since there was no recovery of NE uptake. They hypothesize that noradrenergic neurons that escape the toxic effects of 6OHDA react by increasing their NE-synthesizing capacity. Our results suggest that the compensatory mechanisms that follow destruction of central noradrenergic systems include proliferative changes of dopamine systems. Since tyrosine hydroxylase is common to both catecholaminergic systems, its recovery could be mediated by increased activity or proliferation of dopaminergic terminals.

Compensatory changes such as those described here may be representative of adaptive processes available to neurotransmitter systems throughout the nervous system.

\section{References}

Acheson, A. L., and M. J. Zigmond (1981) Short and long term changes in tyrosine hydroxylase activity in rat brain after subtotal destruction of central noradrenergic neurons. J. Neurosci. 1: 493-504.

Acheson, A. L., M. J. Zigmond, and E. M. Stricker (1980) Compensatory increase in tyrosine hydroxylase activity in rat brain after intraventricular injections of 6-hydroxydopamine. Science 207: 537-540.

Berger, B., J. P. Tassin, G. Blanc, M. A. Moyne, and A. M. Thierry (1974) Histochemical confirmation for dopaminergic innervation of the rat cerebral cortex after destruction of the noradrenergic ascending pathways. Brain Res. 81: 332-337.

Emson, P. C., and G. F. Koob (1978) The origin and distribution of dopamine-containing afferents to the rat frontal cortex. Brain Res. 142: 249-267.

Harik, S. I., J. C. LaManna, A. I. Light, and M. Rosenthal (1979) Cerebral norepinephrine: Influence on cortical oxidative metabolism in situ. Science 206: 69-71.

Harik, S. I., R. B. Duckrow, J. C. LaManna, M. Rosenthal, V. K. Sharma, and S. P. Banerjee (1981) Cerebral compensation for chronic noradrenergic denervation induced by locus ce- ruleus lesion: Recovery of receptor binding, isoproterenolinduced adenylate cyclase activity, and oxidative metabolism. J. Neurosci. 1: 641-649.

Henry, D. P., B. J. Starman, D. G. Johnson, and R. H. Williams (1975) A sensitive radioenzymatic assay for norepinephrine in tissue and plasma. Life Sci. 16: 375-384.

Horn, A. S., J. T. Coyle, and S. H. Snyder (1971) Catecholamine uptake by synaptosomes from rat brain: Structure-activity relationships of drugs with differential effects on dopamine and norepinephrine neurons. Mol. Pharmacol. 7: 66-80.

Kellar, K. J., P. A. Brown, J. Madrid, M. Bernstein, J. VernikosDanellis, and W. R. Mehler (1977) Origins of serotonin innervation of forebrain structures. Exp. Neurol. 56: 52-62.

Kuhar, M. J. (1973) Neurotransmitter uptake: A tool in identifying neurotransmitter-specific pathways. Life Sci. 13: 1623-1634.

Kuhar, M. J., G. K. Aghajanian, and R. H. Roth (1972) Tryptophan hydroxylase activity and synaptosomal uptake of serotonin in discreet brain regions after midbrain raphe lesions: Correlations with serotonin levels and histochemical fluorescence. Brain Res. 44: 165-176.

Kuhar, M. J., V. H. Sethy, R. H. Roth, and G. K. Aghajanian (1973) Choline: Selective accumulation by central cholinergic neurons. J. Neurochem. 20: 581-593.

LaManna, J. C., S. I. Harik, A. I. Light, and M. Rosenthal (1981) Norepinephrine depletion alters cerebral oxidative metabolism in the 'active' state. Brain Res. 204: 87-101.

Lindvall, O., A. Bjorkland, R. Y. Moore, and U. Stenevi (1974) Mesencephalic dopamine neurons projecting to neocortex. Brain Res. 81: 325-331.

Logan, W. J. (1977) Neurotransmitter uptake by normal and transected rat spinal cord. Neurology (N. Y.) 27: 362-363.

Logan, W. J., and S. I. Harik (1981) Locus ceruleus lesion: Acute and long-term effects on high-affinity uptake of norepinephrine, dopamine, serotonin, glutamate, GABA and choline in rat cerebral cortex. Neurology (N. Y.) Part II 31: 165.

Logan, W. J., and S. H. Snyder (1972) High affinity uptake systems for glycine, glutamic and aspartic acids in synaptosomes of rat central nervous tissues. Brain Res. 42: 413-431.

Logan, W. J., and J. M. Swanson (1979) Erythrosin B inhibition of neurotransmitter accumulation by rat brain homogenate. Science 206: 363-364.

Sharma, V. K., S. I. Harik, R. Busto, M. Ganapathi, and S. P. Banerjee (1979) Locus ceruleus lesion and chronic reserpine treatment: Effect on adrenergic and cholinergic receptors in cerebral cortex and hippocampus. Exp. Neurol. 65: 685-690.

Sharma, V. K., S. I. Harik, R. Busto, and S. P. Banerjee (1981) Effects of noradrenaline depletion on adrenergic and muscarinic cholinergic receptors in the cerebral cortex, hippocampus, and cerebellum. Exp. Neurol. 72: 179-194.

Snyder, S. H., and J. T. Coyle (1969) Regional differences in ${ }^{3} \mathrm{H}$-norepinephrine and ${ }^{3} \mathrm{H}$-dopamine uptake into rat brain homogenates. J. Pharmacol. Exp. Ther. 165: 78-86.

Storm-Mathisen, J. (1977) Glutamic acid and excitatory nerve endings: Reduction of glutamic acid uptake after axotomy. Brain Res. 120: 379-386.

Storm-Mathisen, J., and H. C. Guldberg (1974) 5-Hydroxytryptamine and noradrenaline in the hippocampal region: Effect of transection of afferent pathways on endogenous levels, high affinity uptake and some transmitter-related enzymes. J. Neurochem. 22: 793-803.

Tassin, J. P., A. M. Thierry, G. Blanc, and J. Glowinski (1974) Evidence for a specific uptake of dopamine by dopaminergic terminals of the rat cerebral cortex. Naunyn Schmiedebergs Arch. Pharmacol. 282: 239-244.

Zigmond, M. J., J. P. Chalmers, J. R. Simpson, and R. J. Wurtman (1971) Effect of lateral hypothalamic lesions on uptake of norepinephrine by brain homogenates. J. Pharmacol. Exp. Ther. 179: 20-28. 